$\begin{array}{cl}\begin{array}{c}\text { Revue } \\ \text { del'histoire }\end{array} & \text { Revue de l'histoire des religions } \\ \text { des religions } & 4 \text { | } 2005 \\ & \begin{array}{l}\text { Lieux de culte, lieux saints dans le judaïsme, le } \\ \text { christianisme et l'islam }\end{array}\end{array}$

\title{
Un sanctuaire soufi en Inde : le dargâh de Nizamuddin à Delhi
}

A Sufi Shrine in India: Nizamuddin dargâh in Delhi

\section{Marc Gaborieau}

\section{OpenEdition}

\section{Journals}

Édition électronique

URL : http://journals.openedition.org/rhr/4229

DOI : $10.4000 /$ rhr.4229

ISSN : 2105-2573

Éditeur

Armand Colin

Édition imprimée

Date de publication : 1 octobre 2005

Pagination : 529-555

ISBN : 2200-92087-3

ISSN : 0035-1423

Référence électronique

Marc Gaborieau, «Un sanctuaire soufi en Inde : le dargâh de Nizamuddin à Delhi », Revue de l'histoire des religions [En ligne], 4 | 2005, mis en ligne le 18 janvier 2010, consulté le 19 avril 2019. URL : http:// journals.openedition.org/rhr/4229; DOI : 10.4000/rhr.4229 


\section{MARC GABORIEAU}

Centre National de la Recherche Scientifique

École des Hautes Études en Sciences Sociales

\section{Un sanctuaire soufi en Inde : le dargâh de Nizamuddin à Delhi}

Voici la présentation d'un sanctuaire soufi typique de l'Inde, Nizamuddin, bâti autour de la tombe de Nizâmu'd-Dîn Auliyâ (m. 1325) de la confrérie Chishtiyya. Vient d'abord la description de ce centre de pèlerinage. Puis sont analysées les deux sortes de rites exécutés par les fidèles : prières et offrandes pour obtenir les faveurs d'Allah par l'intercession du saint; récitations rythmées (dhikr) et chants mystiques (qawwâlî) qui conduisent à des extases interprétées comme des contacts directs avec Dieu. L'article se clôt sur une étude ethnographique du monde hiérarchique et conflictuel du personnel attaché au sanctuaire : desservants de haut statut et musiciens de basse caste... sans oublier leurs femmes invisibles.

\section{A Sufi Shrine in India: Nizamuddin dargâh in Delhi.}

Here is a presentation of an exemplary Sufi shrine in India, Nizamuddin (Delhi), built around the tomb of Nizâmu'd-Dîn Auliyâ (d. 1325) of the Chishtiyya order. This pilgrimage centre is first described. Then the two kinds of rituals performed by the devotees are analysed: prayers and offerings to get favours from Allah through the intercession of the saint; rhythmical recitations (dhikr) and mystical songs (qawwâlî) which induce ecstasies interpreted as direct contacts with God. Finally comes an ethnographical study of the hierarchical and conflict-ridden world of the shrine's personnel: high status servants of the tomb and low caste musicians... not to forget their invisible wives. 
Les lieux de culte en Islam illustrent deux tendances contradictoires. L'une, universaliste et non controversée, met au premier plan ce qui est commun à l'ensemble de la communauté religieuse et s'illustre de deux façons : d'abord par ces deux grands lieux centraux uniques, buts de pèlerinage par excellence, que sont La Mecque et Jérusalem ; puis au niveau local par les mosquées qui se multiplient à l'infini, mais pour l'exécution d'un rituel codifié et la diffusion d'un enseignement standardisé où tous les musulmans se reconnaissent. L'autre tendance est particulariste et donne lieu à des controverses : elle concentre la dévotion sur des lieux appelés maqâm qui ont été sanctifiés par la présence de saints, et qui sont le plus souvent leurs tombes; ces sanctuaires reflètent les cultures locales et leur héritage préislamique. C'est ce qu'il est convenu d'appeler le culte des saints - improprement d'ailleurs puisque, nous le verrons, les dévotions ne s'adressent pas directement à eux.

Cet article étudie un lieu sacré de ce second type à Delhi, la grande capitale historique de l'islam en Inde : il s'est développé autour de la tombe du plus important des saints de la confrérie soufie Chishtiyya, le mystique Nizâmu'd-Dîn Auliyâ (c. 1243-1325). Son nom, sous la forme anglo-indienne de Nizamuddin, désigne aussi trois réalités emboîtées : le complexe religieux proprement dit ; la petite agglomération alentour, autrefois fortifiée, qui abrite les desservants qui passent pour les descendants de la sœur du saint (mort célibataire) et leurs serviteurs ; enfin le quartier moderne de New Delhi qui les englobe.

Nous présenterons d'abord le saint et le complexe qui entoure sa tombe, complexe que, pour des raisons que nous expliquerons plus loin, nous appellerons sanctuaire au sens que le Petit Robert donne à ce terme : «Édifice consacré aux cérémonies d'une religion ; lieu saint $»^{1}$. Mais il faudra garder présent à l'esprit que sous ce terme, nous ne désignerons pas seulement un seul édifice, le mausolée qui entoure la tombe, mais le complexe des bâtiments qui s'étalent autour de lui et des espaces de circulation qui s'insèrent entre eux. Cet ensemble qui forme un tout est aussi parfois appelé hospice, couvent,

1. Petit Robert, éd. 2000, p. 2275, sens n 2 . 
ou monastère ; nous avons évité ces termes à cause de leur connotation chrétienne, et parce qu'ils suggèrent une vie monastique, alors qu'à Nizammuddin les desservants, mariés, ne sont pas des moines, et que l'accent est mis sur le pèlerinage et sur les rites et pratiques mystiques qui l'accompagnent.

Nous nous demanderons de quelle façon et par quels intermédiaires ce lieu rendu sacré par la présence d'un saint facilite aux pèlerins l'accès à Allah et à ses faveurs. Cette interrogation prendra la forme d'une double série de questions. Les premières concernent les fonctions religieuses du sanctuaire : comment y approche-t-on Dieu ? Est-ce de façon médiate par des rites qui constitueraient une sorte de liturgie ? Ou y a-t-il des pratiques mystiques qui permettent d'approcher plus immédiatement le divin ? Ou y a-t-il un dosage des deux? Nous nous demanderons en second lieu comment s'organise la société complexe et hiérarchisée de ceux qui assurent le service du sanctuaire, c'est-à-dire les desservants (khâdim, pl. khuddâm) et ceux qui sont à leur service. En conclusion, après avoir souligné les spécificités de Nizamuddin, nous nous interrogerons sur son rayonnement dans le contexte indien et dans le monde.

Cette étude doit beaucoup à ses devanciers, même si j'ai moi-même beaucoup fréquenté Nizamuddin depuis une trentaine d'années. Mon travail s'inscrit d'abord dans la continuité des premiers travaux sur l'islam indien qui ont été inaugurés en France par Garcin de Tassy (1794-1878) dès 1831, avec précisément un opuscule toujours d'actualité sur le culte des saints dans lequel il décelait des «particularités » indiennes ${ }^{2}$. Ils ont été poursuivis jusqu'à nos jours par de nombreux chercheurs de notre pays et des autres pays occidentaux, sans compter l'immense production en persan, en ourdou et en anglais qui s'est développée dans le sous-continent indien avec des

2. Garcin de Tassy, Mémoire sur quelques particularités de la religion musulmane en Inde d'après les ouvrages hindoustanis, Paris, Journal asiatique, puis Imprimerie nationale, 1831 ( $2^{\mathrm{e}}$ éd. rev., Paris, Labitte, 1869); sur cet opuscule voir Marc Gaborieau, "Muslim Saints, Faquirs, and Pilgrims in 1831 according to Garcin de Tassy", in Jamal Malik (éd.), Perspectives of Mutual Encounters in South Asian History, 1760-1860, Leyde, E. J. Brill, 2000, p. $128-156$. 
synthèses anciennes ou récentes ${ }^{3}$, et de remarquables monographies publiées depuis près de vingt-cinq ans ${ }^{4}$. Je m'y suis moi-même intéressé dès mes premiers travaux sur le Népal et le Tibet, pour faire la description ethnologique de ce culte des saints en milieu rural et urbain, et pour jauger les implications théologico-juridiques des controverses qu'il suscite 5 .

Le choix de Nizamuddin parmi les multiples exemples qui ont été étudiés se justifie pour deux raisons : l'importance historique,

3. John A. Subhan, Sufism, its Saints and Shrines. An Introduction to the Study of Sufism with special Reference to India, Lahore, 1938 (réimpression New York, Samuel Wiser, 1958) ; Christian W. Troll (éd.), 1989, Muslim Shrines in India. Their Character, History and Significance, Delhi, Oxford University Press, 1989 ( $2^{2}$ éd. 2003, avec une introduction de Marc Gaborieau); Henri Chambert-Loir \& Claude Guillot (éd.), Le culte des saints dans le monde musulman, Paris, École Française d'Extrême Orient, 1995 (voir en particulier les articles sur le Pakistan, l'Inde et le Bangladesh par Denis Matringe, Marc Gaborieau, Catherine Champion et Samuel Landell-Mills, p. 167-227) ; Marc Gaborieau, «Il ruolo del sufismo e delle confraternite musulmane nell'India contemporanea », in Marietta Stepanyants (éd.), Sufismo e confraternite nell'islam contemporaneo. Il difficile equilibrio tra mistica e politica, Turin, Fondation Giovanni Agnelli, 2003, p. 71-101.

4. Richard M Eaton, "Court of Man, Court of God. Local Perceptions of the Shrine of Bâbâ Farîd, Pakpattan, Punjab", in Richard C. Martin (éd.), Islam in Local Contexts, vol. 17 de Contributions to Asian Studies, Leiden, E. J. Brill, 1982, p. 44-61, ainsi que "The Political and Religious Authority of the Shrine of Bâbâ Farîd", in Barbara D. Metcalf (éd.), Moral Conduct and Authority. The Place of adab in South Asian Islam, Berkeley, University of California Press, 1984, p. 333-356 ; P. M. Currie, The Shrine and Cult of Mu'în al-Dîn Chishtî of Ajmer, Delhi, Oxford University Press, 1992 ; Carl W. Ernst, Eternal Garden. Mysticism, History, and Politics at a South Asian Sufi Center, Albany, State University of New York Press, 1992 (2e éd., Delhi, Oxford University Press, 2004) ; Claudia Liebeskind, Piety on its Knees. Three Sufi Traditions in South Asia in Modern Times, Delhi, Oxford University Press, 1998 ; Soren Lassen, "A meeting Place of Traditions. Ritual Practice and Leadership in an Islamic Religious Institution in South India”, Thèse inédite, Université de Copenhague, 2004.

5. Marc Gaborieau, "A Nineteenth-Century Indian 'Wahhabi' Tract Against the Cult of Muslim Saints: Al-Balâgh al-Mubin", in Christian W. Troll, Muslim Shrines in India. Their Character, History and Significance, Delhi, Oxford University Press, 1989, p. 198-239 (2e éd. 2003) ; Marc Gaborieau, « Le culte des saints musulmans en tant que rituel : controverses juridiques », Archives de sciences sociales des religions, $\mathrm{n}^{\circ} 85$, janvier-mars 1994, p. 85-98 ( ${ }^{\circ}$ spécial "Oubli et remémoration des rites. Histoire d'une répugnance », éd. par John Scheid \& Francis Schmidt). 
politique et culturelle de ce lieu de pèlerinage situé dans la grande capitale de l'islam indien ne s'est jamais démentie ; c'est le sanctuaire qui a suscité le plus large éventail disciplinaire de recherches allant de l'archéologie et de l'histoire jusqu'à la musicologie, en passant par l'islamologie, l'anthropologie et la littérature, comme on le constatera au fil des notes de cet article. C'est indubitablement le foyer le mieux connu de l'islam indien; il peut servir de base pour en définir les traits spécifiques. Les analyses qui suivent ont fait l'objet d'une présentation plus circonstanciée dans mon séminaire de l'EHESS sur l'islam indo-pakistanais pendant deux ans, de 2000 à $2002^{6}$.

\section{LE SAINT EN SON SANCTUAIRE}

Le sanctuaire s'est développé à partir de rien autour de la tombe de Nizâmu'd-Dîn, qui a été enterré en 1325 dans un lieu désert, luimême à quelque distance de son ermitage ; l'un et l'autre endroits étant alors situés dans la campagne à bonne distance au Nord des trois capitales déjà bâties depuis un peu plus d'un siècle par les premiers sultans de Delhi. Ce lieu devint rapidement un important foyer religieux, politique et culturel en raison de la stature du personnage qui y était inhumé.

Nizâmu'd-Dîn Auliyâa, la confrérie Chishtiyya et la culture indo-persane

Un saint musulman, techniquement désigné par le terme arabe walî, est généralement appelé en Inde pîr, terme persan signifiant littéralement un vieillard; comme son équivalent arabe shaikh, ce terme peut désigner soit un saint mort déjà vénéré, soit un maître soufi encore vivant. Il s'appliquera donc aussi, nous le verrons, à ses successeurs (souvent apparentés) qui desservent sa tombe.

Nizâmu'd-Dîn appartenait à une confrérie d'origine afghane, la Chishtiyya, qui a été la plus spécifiquement indienne, et qui est restée à travers le sultanat de Delhi (1210-1526) et l'empire moghol

6. Je remercie mes auditeurs pour leurs commentaires et leurs critiques qui m'ont permis de préciser mes analyses. 
(1526-1765) l'ordre tutélaire de la majorité des souverains musulmans de l'Inde ; c'est aussi la confrérie indienne la mieux connue ${ }^{7}$. Nizâmu'd-Dîn en est aussi le premier maillon important qui soit connu par des sources historiques et hagiographiques indo-persanes contemporaines, en particulier le recueil de ses « dits » (malfûzât) qui ont été édités de son vivant sous sa supervision ${ }^{8}$. Il est le parfait exemple du cheikh soufi du sultanat indien ${ }^{9}$ formé sur le modèle de l'Asie centrale : il combine la culture persane et la sensibilité poétique avec des dons extatiques et des pouvoirs miraculeux ; généreux avec ses disciples fidèles, il peut être terrible avec ses ennemis ; il vit ostensiblement dans son ermitage à l'écart du sultan, mais assez près de la cour pour influer sur elle et sur les courtisans qui sont souvent ses familiers ; méprisant ostensiblement le pouvoir, il est conscient néanmoins d'influer sur lui, tandis que le sultan a besoin de lui pour asseoir sa légitimité.

Le familier le plus assidu de Nizâmu'd-Dîn était le grand Amîr Khusrau (1253-1325), poète de cour protégé des sultans, mais aussi disciple préféré de Nizâmu'd-Dîn aux pieds de qui il fut enterré : le saint et le poète fixèrent pour des siècles les standards de la culture indo-persane, combinant un haut niveau de prose et de poésie persane, une sensibilité soufie, et un intérêt certain pour la littérature et les coutumes locales : ils célébraient en particulier, comme on le verra, la fête hindoue de basant.

\section{Le sanctuaire}

Pour désigner ce type d'institution il existe en Inde de multiples termes qui connotent leur nature et surtout leur importance : mazâr, littéralement « lieu de visite », ne convient qu'à une modeste tombe ;

7. Carl W. Ernst \& Bruce B. Lawrence, Sufi Martyrs of Love. The Chishti Sufi Order in South Asia and Beyond, New York, Palgrave MacMillan, 2002.

8. Bruce B. Lawrence, Morals for the Heart. Conversations of Shaykh Nizam ad-din Awliya recorded by Hasan Sijzi, translated by Bruce B. Lawrence; introduction by Khaliq Ahmad Nizami, New York, Paulist Press, 1992.

9. Simon Digby, "The Sufi Shaikh as a Source of Authority in Mediaeval India", in Marc Gaborieau (éd.), Islam et société en Asie du Sud, Paris, EHESS, 1986 (Collection « Purushârtha », n 9), p. 57-77. 
l'arabe takya (en turc tekke) et le persan khânqâh ont en Inde le sens d'hospice ou de monastère, respectivement de petite et de grande taille, impliquant la présence permanente de soufis ; le second est applicable à Nizâmuddin ; enfin, plus honorifiques, les termes persans astâna, et plus fréquemment dargâh - qui signifient littéralement le «seuil » de la maison d'un supérieur - désignent par métonymie l'ensemble du sanctuaire comme objet de respect. Nous sommes ici, en effet, dans le registre du vocabulaire impérial : le saint est le véritable sultan de l'Inde; son sanctuaire est traité comme un « palais », car c'est ainsi qu'il faut traduire le terme dargâh qui est couramment employé pour désigner Nizamuddin et les sanctuaires similaires; nous le franciserons en dargâh.

Pour le visiteur qui arrive aujourd'hui en taxi par les grandes avenues modernes et doit continuer sa route à pied, le complexe de Nizamuddin apparaît comme un vaste enchevêtrement de ruelles, à travers les maisons et les monuments ; c'est une cour des miracles où se côtoient desservants, musiciens, pèlerins, mendiants et malades plus ou moins agités. On finit par arriver à un espace central plus dégagé, où se détachent une mosquée du XIV $\mathrm{Xv}^{\mathrm{e}}$ siècle de grès rouge rehaussé d'incrustations de marbre blanc, et le mausolée de marbre blanc du saint construit à l'époque moghole et plusieurs fois restauré.

Depuis les premiers relevés britanniques du milieu du XIX ${ }^{\mathrm{e}}$ siècle jusqu'à nos jours un inventaire minutieux des monuments qui sont généralement datés permet de reconstruire l'histoire du dargâh ${ }^{10}$. L'ensemble était autrefois fortifié : il était entouré d'un mur percé de plusieurs portes, avec une citadelle au Sud-Ouest.

La porte principale, datée du XIV siècle, se trouve au Nord. Après l'avoir franchie, en se dirigeant droit vers le Sud, le visiteur accède immédiatement aux principaux édifices rituels. D'abord s'ouvre un

10. M. Zafar Hasan, A Guide to Nizamu-d-Din, Calcutta, Memoirs of the Archeological Survey of India, ${ }^{\circ} 10,1922$. Pour des synthèses voir J. BurtonPage, "Dihlî”, Encyclopaedia of Islam, 2e éd. (version anglaise), vol. II, 1965, p. 255-266, particulièrement p. 261-263 et fig. 5 ; Y. D. Sharma, Delhi and its Neighbourhood, New Delhi, Archeological Survey of India, 2e éd., 1974, p. 115-121. Pour une étude récente des monuments voir Catherine B. Asher, Architecture of Mughal India, Cambridge, Cambridge University Press, 1992, à l'index sous "Delhi, Dargah Nizam al-Din". 
très large puits où une volée de marches permet de descendre jusqu'au niveau de l'eau : cet édifice, désigné par le terme hindi bâolî, ne servait pas seulement pour les ablutions ; comme dans de nombreux sanctuaires soufis, son eau est réputée avoir des vertus miraculeuses. On débouche ensuite sur la cour principale du dargâh, avec sur la droite la grande mosquée du XIV e, orientée vers La Mecque, c'est-àdire vers l'Ouest, ouvrant donc vers l'Est.

Juste à l'est de la mosquée se trouve le plus grand espace ouvert, au milieu duquel s'élève le mausolée du saint construit sur une plateforme à laquelle on accède par plusieurs marches. Il comprend une salle funéraire carrée surmontée d'un dôme ; on y pénètre par une porte s'ouvrant au Sud, au pied de la tombe qui est orientée nord-sud, car les musulmans sont inhumés en Inde couchés sur le dos, la tête au Nord, le visage reposant sur le côté droit, tourné vers La Mecque ; l'espace est dégagé pour permettre au pèlerin de faire le tour de la tombe. Une véranda reposant sur des piliers entoure la chambre funéraire. L'espace au Sud de la tombe, face à sa porte d'entrée, est largement dégagé pour faciliter l'accès des fidèles et les rassemblements des desservants et des musiciens lors des fêtes. Un peu plus loin au Sud se trouve l'autre tombe importante pour le rituel, celle d'Amîr Khusrau.

Le reste du complexe a, de façon enchevêtrée, cinq fonctions principales. C'est d'abord un immense cimetière où sont enterrés non seulement des descendants de la sœur du saint, mais surtout des aristocrates, spécialement des dignitaires et des princes de la dynastie moghole dont les tombes selon les époques sont des mausolées monumentaux ou de modestes enclos; ces nobles personnages recherchaient la baraka du saint pour s'assurer un repos éternel. La seconde fonction relève du domaine spirituel : les desservants (sing. khâdim, pl. khuddâm), qui jouent aussi le rôle de guides spirituels ou d'exorcistes, reçoivent leurs visiteurs dans des cellules (hujra) disposées sur le pourtour des tombes. La troisième fonction est charitable : le dargâh entretient sur les dons des fidèles une cuisine, désignée par le terme persan langar, qui distribue tous les jours aux nécessiteux des repas gratuits. La quatrième fonction relève du commerce des biens matériels: des boutiques, appartenant aux 
familles des desservants, vendent aux pèlerins des fleurs et de l'encens pour offrir sur la tombe du saint, et des livres et des images pieuses pour leur édification ; un dispensaire de médecine yûnânî (la médecine grecque adoptée par l'islam) est à disposition des malades. Enfin, sur le pourtour, sont disposées les habitations des desservants, où sont consignées leurs femmes qui ne fréquentent pas les espaces ouverts du sanctuaire; les desservants y logent éventuellement les pèlerins de marque qui se montrent généreux à leur égard. Les serviteurs des desservants, porteurs d'eau et musiciens, logent aussi dans cet espace.

Sorte de ville dans la ville, le dargâh est monde clos qui se referme sur ses multiples fonctions liées à la baraka du saint; nous allons maintenant les détailler avant de nous interroger, dans la troisième partie de cet article, sur les hiérarchies qu'elles génèrent.

\section{DES SAINTS À ALLAH : RITES ET EXPÉRIENCES MYSTIQUES}

Du point de vue religieux, le dargâh est un lieu d'intercession (shafâ 'at) : la baraka, la grâce d'origine divine, qui imprègne le saint et le sanctuaire, rejaillit sur les fidèles et leur bien-être matériel et spirituel. Le saint joue donc le rôle d'intermédiaire entre Allah et les pèlerins. Mais de quelle façon? On oppose souvent le rituel canonique de la mosquée, où l'accès au divin est médiatisé par des paroles et des gestes, et l'itinéraire mystique qui donne une intuition immédiate du divin par l'extase. Ce qui est frappant dans un dargâh, c'est que les deux voies vers Dieu coexistent. À Nizamuddin, il n'y a pas, d'un côté, un rituel médiat dont le lieu exclusif serait la mosquée et, de l'autre, une expérience mystique non médiatisée qui se ferait dans le mausolée et la cour ouverte qui s'étend devant sa porte. Non, dans l'espace mystique ainsi délimité - c'est-à-dire le mausolée et la cour qui lui fait face - rites et expérience mystique coexistent.

\section{Les rites}

Dans l'histoire de l'islam ce sont les adversaires du soufisme et du culte des saints, les « ultra-orthodoxes », qui ont attiré l'attention 
sur la nature rituelle des dévotions qui se déroulent sur les tombes des saints, comme je l'ai montré en détail ailleurs ${ }^{11}$. L'argumentaire n'est pas nouveau ${ }^{12}$ : il a été fixé au tournant des XIII ${ }^{\mathrm{e}}$ et XIV ${ }^{\mathrm{e}}$ siècles par le juriste et polémiste damascène Ibn Taimiyya (1263-1328) ${ }^{13}$ et popularisé au XVIII ${ }^{\mathrm{e}}$-XIX ${ }^{\mathrm{e}}$ siècles par les Wahhabites d'Arabie et par le Yéménite Muhammad 'Alî al-Shawkânî (1760-1834) ${ }^{14}$; il a été repris à satiété en Inde par les réformateurs du XIX et du XXe siècle qui se réclament de l'héritage du grand soufi charismatique et jihadiste Sayyid Ahmad Barelwî (1786-1831) ${ }^{15}$ et sont regroupés aujourd'hui dans les écoles de Deoband et des Ahl-i Hadîth. Il consiste à montrer de façon tout à fait perspicace pourquoi ces dévotions ne peuvent être définies que comme des rites du point de vue de la jurisprudence, science reine de l'islam, comme de la théologie.

Ces dévotions se caractérisent d'abord par le lieu et par le temps. Il faut venir prier sur la tombe, lieu imprégné de la baraka du saint ; il s'agit donc d'un pèlerinage. Il y a ensuite une périodicité de ces visites : les plus dévots peuvent se rendre sur la tombe tous les

11. Marc Gaborieau, «Le culte des saints musulmans en tant que rituel : controverses juridiques », Archives de sciences sociales des religions, $\mathrm{n}^{\circ} 85$, janvier-mars 1994, op. cit., p. 85-98.

12. Marc Gaborieau, «Tarîqa et orthodoxie », p. 195-202 et (avec Nicole Grandin) «Le renouveau confrérique (fin XVIII ${ }^{\mathrm{e}}-\mathrm{XIX}$ siècle) », p. 68-83, dans Alexandre Popovic \& Gilles Veinstein (éd.), Les voies d'Allah. Les ordres mystiques dans le monde musulman des origines à aujourd'hui, Paris, Fayard, 1996.

13. Muhammad Umar Memon, Ibn Taimîya's Struggle against Popular Religion, Paris/La haye, Mouton, 1976 ; Niels Henrik Olesen, Culte des saints et pèlerinage chez Ibn Taymiyya, Paris, Geuthner, 1991.

14. Bernard A. Haykel, "Order and Righteousness: Muhammad `Alî alShawkânî and the Nature of the Islamic State in Yemen", Thèse inédite, Oxford, Faculty of Oriental Studies, 1997, p. 156-171 (À paraître à Cambridge University Press).

15. Marc Gaborieau, "A Nineteenth-Century Indian 'Wahhabi' Tract Against the Cult of Muslim Saints...", op. cit ; Marc Gaborieau, «Les débats sur l'acculturation chez les musulmans indiens au début du XIX siècle », dans Jackie Assayag \& Gilles Tarabout (éd.), Altérité et identité. Islam et christianisme en Inde, Paris, EHESS, 1997, p. 221-237 (Collection « Purushârtha », $\mathrm{n}^{\circ} 19$ ) ; Marc Gaborieau, "Criticizing the Sufis: The Debate in Early-NineteenthCentury India", in Frederick De Jong \& Bernd Radtke (éd.), Islamic Mysticism Contested. Thirteen Centuries of Controversies and Polemics, Leyde, Brill, p. $452-467$. 
jours, le crépuscule du soir étant le moment le plus favorable ; il y a un premier temps fort chaque semaine, le jeudi soir, en fait la vigile du vendredi, jour sacré de la grande prière. Mais les véritables sommets de la dévotion sont les quatre grandes fêtes annuelles ${ }^{16}$ qui attirent des dizaines, voire des centaines de milliers de fidèles venus de tout le sous-continent indien, Pakistan et Bangladesh compris. Trois sont proprement islamiques : elles marquent selon le calendrier purement lunaire l'anniversaire de la mort de Nizâmu'd-Dîn, d'Amîr Khusrau et du Prophète Muhammad ; elles sont appelées «noces » ('urs), car la mort est l'union mystique définitive de l'âme humaine féminisée avec Dieu. La quatrième, dont la date est fixée selon le calendrier luni-solaire de l'Inde, est une fête hindoue de basant qui marque le début des réjouissances de printemps ${ }^{17}$.

Les rites se marquent d'abord par des gestes. Le pèlerin doit entrer dans le mausolée (du moins s'il est un homme, car les femmes doivent rester à l'extérieur, dans la partie sud de la véranda à la hauteur de la tête du saint). Il en fait le tour dans le sens des aiguilles d'une montre en gardant toujours la tombe à sa droite ; il touche cette dernière de la main ou du front. Il y dépose des offrandes qui doivent être à tout le moins des fleurs, mais qui peuvent plus substantielles sous la forme d'argent et d'objets précieux ; les plus riches financent le riche drap brodé qui recouvre la tombe et est changé lors de la fête annuelle, ou font de généreuses donations aux desservants.

16. Patricia Jeffery, "Creating a Scene: The Disruption of Ceremonial in a Sufi Shrine", in Imtiaz Ahmad (éd.), Ritual and Religion among Muslims in India, Delhi, Manohar, 1981, p. 162-194.

17. Cette fête, célébrée le cinquième jour de la quinzaine claire du mois lunaire hindou de mâgha (janvier-février), marque le début des réjouissances de printemps qui dureront six semaines jusqu'à la seconde fête de printemps, holî, qui s'étend sur la dernière semaine de la quinzaine claire du mois de phâlguna (février-mars). Sur la place de ces festivités de printemps dans le calendrier, voir Marc Gaborieau, «Les fêtes, le temps, l'espace : structure du calendrier hindou dans sa version indo-népalaise », L'Homme, XXII, 3, 1982, p. 11-29 et particulièrement p. 17-18; pour les célébrations en contexte indien, voir R. P. Tripâthî, Hinduon-ke vrata, parva aur tyauhâr [Hindi : " Jeûnes, fêtes et festivals des hindous »], Allahabad, Lokbhâratî Prakâshan, 1971, p. 305-307. 
Des paroles accompagnent les gestes. Elles ont une forme canonique, appelée fâtiha, du nom de la première sourate du Coran : elles s'adressent directement à Dieu au nom du saint, en Lui demandant de faire retomber le mérite de ces offrandes sur les fidèles. Ce respect de la forme canonique n'empêche pas le pèlerin de faire un marché avec le saint sous la forme d'un vœu (nadhr) : «Si j'obtiens telle ou telle faveur, je reviendrai avec telle ou telle offrande ».

Enfin le départ est religieusement marqué. Le pèlerin doit sortir du mausolée à reculons, pour ne pas montrer son dos au saint. Il ramène chez lui des objets qui ont été en contact avec la tombe ou la terre du sanctuaire, et sont donc imprégnés de la baraka du saint; d'où le nom de ces « reliques » : tabarruk.

\section{Controverses sur l'interprétation des rites}

Les ultra-orthodoxes ont voulu - sans grand succès, sauf en Arabie Saoudite - interdire ces rites sur les tombes de saints. Leurs arguments sont-ils valides ? Ils ont à juste titre, du point de vue juridique, souligné la nature rituelle de ces dévotions définies par la combinaison d'un temps, d'un lieu, de gestes et de paroles standardisées; et en cela ils sont d'accord avec les partisans du culte des saints. Là où les deux camps divergent, c'est sur la licéité de ces rites. Le consensus islamique avait, dès le $\mathrm{XI}^{\mathrm{e}}$ siècle, accepté ces dévotions sur les tombes des saints et fait de leurs miracles un article de foi. Au nom de la pureté de l'islam des origines qui ignorait encore les saints, les ultra-orthodoxes maintiennent que ces rites ne peuvent être célébrés que pour Dieu : seuls deux pèlerinages, La Mecque et Jérusalem, sont licites ; même celui du tombeau du Prophète à Médine est rejeté. On ne peut tourner qu'autour de la Ka 'ba à La Mecque, aucun autre monument n'a droit à cet honneur. Les rites sur les tombes des saints sont rejetés car ils sont une offense à la majesté et à l'exclusivité divine.

Une argumentation théologique conforte cette condamnation légaliste. Faire ces rites, disent les ultra-orthodoxes, revient à donner à un personnage humain, à un saint, des pouvoirs qui n'appartiennent qu'à Dieu. Lui seul peut faire des miracles ; Lui seul peut exaucer 
les prières. Le saint est impuissant à exaucer les fidèles. Il n'a même aucun pouvoir d'intercession: Dieu accorde ses faveurs à qui Il veut et quand Il veut; personne ne peut influer sur Lui. Le seul homme qui aura jamais un pouvoir d'intercession sera le Prophète Muhammad, encore ne l'exercera-t-il qu'au jour du Jugement dernier, et seulement avec la permission d'Allah. Toute la liturgie effectuée sur les tombes des saints est condamnée sans appel, car elle accorde au saint des honneurs qui sont exclusivement réservés à Dieu.

La critique des ultra-orthodoxes est cependant biaisée. Ils caricaturent nettement les dévotions des fidèles en prétendant que leurs dévotions s'adressent exclusivement aux saints, alors que, dans l'esprit des fidèles instruits, le saint n'est qu'un intermédiaire ; de façon ultime, les dévotions s'adressent à Dieu. C'est ce qu' avait bien compris Garcin de Tassy. Les missionnaires protestants en Inde au début du XIX ${ }^{\mathrm{e}}$ siècle ont repris les critiques des ultra-orthoxes contre le culte des saints musulmans, ce qui n'a rien d'étonnant, puisque leurs arguments ont de grandes analogies - sans aucun lien historique - avec les objections calvinistes à la vénération des saints catholiques. Garcin de Tassy au contraire s'enthousiasma pour les dévotions des musulmans à leurs saints : il soulignait dès son Mémoire de 1831 l'analogie entre le culte des saints musulmans et celui des saints du catholicisme :

«Mais ces fatihas (fâtiha) ne s'adressent pas précisément aux saints ; on ne saurait mieux les comparer qu'aux collectes de la messe des fêtes catholiques en l'honneur des saints, où on ne les prie jamais directement. Ainsi, malgré la grande dévotion qu'ont envers leurs saints les musulmans de l'Inde, on ne peut pas dire qu'ils leur adressent directement des prières. ${ }^{18}$ »

Il n'en resta pas moins que les croyances et les pratiques des pèlerins s'écartent des normes des ultra-orthodoxes : ils croient à la baraka attachée aux lieux fréquentés par les saints et à l'intercession de ces derniers ; ils n'hésitent même pas, notamment lors des vœux, à s'adresser directement à eux et à marchander leurs faveurs.

18. Garcin de Tassy, Mémoire sur quelques particularités de la religion musulmane en Inde..., op. cit., $2^{2}$ éd., p. 26 ; pour une analyse détaillée de cette question voir Marc Gaborieau, "Muslim Saints, Faquirs, and Pilgrims in 1831...", op. cit., p. 153-156. 


\section{La voie mystique}

Dans les rites, Allah n'est accessible qu'indirectement; sa présence n'est pas palpable. Il en va autrement si l'on s'engage sur le chemin de l'expérience mystique qui est accessible de deux façons : la voie longue de l'initiation, et la voie courte de l'extase artistique.

Une minorité de pèlerins ne se contente pas des rites, ni de la musique ; ils se choisissent un guide parmi les desservants qui sont aussi des cheikhs soufis, des pîr dans la terminologie indo-persane. Devenus ainsi disciples, ou murîd, ils entreprennent sous sa direction une longue éducation mystique qui, si elle réussit, leur permet dans des cérémonies comme le dhikr d'accéder à des états seconds, à des sortes de transes, où ils se fondent dans le divin, et de développer des pouvoirs surnaturels ${ }^{19}$. Sans percer tous les secrets de l'initiation, une récente monographie a mis en scène les principaux aspects de cette démarche ésotérique qui n'est pas immédiatement observable par le visiteur ${ }^{20}$.

\section{L'extase poétique et musicale}

Plus spectaculaire et moins longue est la voie artistique, observable presque quotidiennement, en tout cas les jeudis soirs et lors des grandes fêtes. Elle requiert, sous la direction des desservants qui sont les maîtres de cérémonie, la présence de musiciens spécialisés de bas statut, les qawwâl, terme d'origine arabe qui signifie celui qui parle, qui récite un texte, car la base de leurs performances musicales est constituée de poésies mystiques originellement en persan, de plus en plus souvent complétées par des compositions dans les langues vernaculaires de l'Inde du Nord. Ces poésies sont chantées avec l'accompagnement d'un harmonium

19. Patricia Jeffery, "Creating a Scene : The Disruption of Ceremonial in a Sufi Shrine', op. cit., p. 189-190.

20. Desiderio Pinto, Piri-Muridi Relationship: A Study of the Nizamuddin Dargah, Delhi, Manohar, 1995. 
pour soutenir la hauteur de la voix, et de plusieurs tambours pour marquer le rythme ${ }^{21}$.

Les chanteurs sont disposés en groupe sur plusieurs lignes parallèles à la façade sud du mausolée, à l'extrémité méridionale de la cour. Les desservants qui patronnent la cérémonie sont disposés dans l'entre deux, sur deux lignes perpendiculaires à la fois à la façade sud du mausolée et aux lignes de chanteurs. Les auditeurs sont assis tout autour.

La séance musicale, ou qawwâlî, ${ }^{22}$ commence par la louange de Dieu, l'éloge du Prophète et de son gendre 'Alî. Elle enchaîne sur des poèmes illustrant les étapes de la quête mystique. Les images et les sentiments évoqués, qui mobilisent la vaste culture mystique de l'auditoire, se combinent à l'effet lancinant de l'exécution musicale et de la danse dans laquelle se lancent de nombreux auditeurs pour induire chez les plus sensibles d'entre eux un état extatique (hâl), qui est interprété comme la rencontre (wajd) avec Allah, sa fusion avec Lui. Ces états mystiques, préfiguration de la mort qui sera l'union définitive avec Dieu, sont hautement valorisés dans la tradition de la confrérie Chishtiyya : le ton a été donné par le saint patron de Delhi, Qutb al-Dîn Bakhtiyâr Kâkî (m. c. 1235), qui mourut à la suite de quatre jours d'extase ininterrompue consécutive à une qawwâlî, passant ainsi sans transition de l'union temporaire à Dieu dans ce monde à l'union définitive dans l'au-delà ; son exemple a été imité jusque dans le $\mathrm{XX}^{\mathrm{e}}$ siècle ${ }^{23}$.

Ainsi donc, par la médiation du rituel, ou plus intuitivement par l'extase, le pèlerin prend appui sur la tombe, le saint qui l'habite et

21. Regula Burkhardt Qureshi, Sufi Music of India and Pakistan: Sound, Context and Meaning in Qawwali, Cambridge, Cambridge University Press, 1986 ( 2 e éd., Chicago, The University of Chicago Press, 1995, with a new preface (p. XII) and one CD of 11 pieces recorded at Nizamuddin) ; Claire Devos, 1995, Qawwali. La musique des maîtres du soufisme, Paris, Éditions du Makar, 1995.

22. Denis Matringe, «Une séance de Qavvâlî archétypale : introduction, transcription et traduction », dans Annie Montaut (éd.), Le Rajasthan. Ses dieux, ses héros, son peuple, Paris, Publications Langues'O, 2000, p. 121-154 ; Nusrat Fateh Ali Khan, Nusrat Fateh Ali Khan en concert à Paris, coffret de trois disques compacts OCORA-Radio France, C 559 072, 073, 074, 1989 ; livret avec traduction des textes par Denis Matringe.

23. Simon Digby, "The Sufi Shaikh as a Source of Authority...", op. cit., p. 61. 
ses desservants pour accéder aux faveurs divines, ou mieux pour s'unir temporairement à Dieu dans une préfiguration de l'au-delà.

\section{LE PERSONNEL DU DARGÂH : HIÉRARCHIES ET CONFLITS}

Le saint, intercesseur par excellence, n'est pas le seul intermédiaire : entre lui et le pèlerin s'intercalent d'autres intermédiaires obligés : les desservants du sanctuaire, qui sont distingués selon la « caste » et le genre. Comment cette microsociété s'organise-t-elle? Les hiérarchies qui s'y dessinent sont-elles exemptes de conflits?

\section{Une société de « castes»}

Le petit monde de Nizamuddin est souvent décrit dans la trop vague phraséologie anglo-indienne comme « féodal ${ }^{24}$ : ce terme suffit-il pour caractériser la stricte hiérarchie qui y règne ? Les écarts de statuts sont en effet très rigidement marqués.

Au plus haut niveau se placent les descendants du saint : je les ai jusqu'ici appelés vaguement desservants (khâdim) du dargâh ; on les désigne collectivement sous le vocable ourdou de pîr-zâde, c'est-à-dire de « descendants de saint ». Ces Pîrzâde, comme nous les appellerons désormais sous une forme francisée, ont fait l'objet de la remarquable enquête ethnologique de Patricia Jeffery ${ }^{25}$. Ils en sont en fait les véritables maîtres et propriétaires du Dargâh. Nizamuddin, qui de façon exceptionnelle dans le monde soufi était resté célibataire, n'a pas laissé d'enfants : les Pîrzâde passent pour descendre de sa sœur et sont traditionnellement divisés en quatre clans rivaux dont l'un s'est éteint au cours du XIX siècle. Dans les années soixante-dix, l'ensemble des Pîrzâde, femmes et enfants compris, représentait plusieurs centaines de personnes. S'affirmant

24. Le terme est employé par Regula Burkhardt Qureshi, Sufi Music of India and Pakistan, op. cit.

25. Patricia Jeffery, Frogs in a Well : Indian Women in Purdah, Londres, Zed Press, 1979. Patricia Jeffery, "Creating a Scene: The Disruption of Ceremonial in a Sufi Shrine", op. cit. 
descendants du Prophète, ils entrent dans la classe privilégiée des « nobles » ou ashrâf, réputés d'origine étrangère, qui occupent le sommet de la société musulmane en Inde ${ }^{26}$. Ils restent très largement endogames préférant le mariage dit «arabe» avec les filles du lignage ; les rares unions externes restent cantonnées à des lignages «nobles ». Le principal symbole de leur statut est la stricte ségrégation des femmes qui - nous y reviendrons - restent cloîtrées chez elles dans les maisons aveugles et le dédale des ruelles qui entourent la tombe. Ils monopolisent les principales fonctions religieuses et économiques du sanctuaire. Selon un système de rotation complexe (qu'on retrouve aussi dans certains sanctuaires hindous), chaque famille assure à tour de rôle l'accueil des pèlerins et reçoit leurs offrandes qui constituent leurs honoraires. En plus de ce service organisé impersonnel, chaque Pîrzâde peut se constituer une clientèle personnalisée de riches pèlerins selon le système dit wakîl-mehmân (littéralement : avocat-invité), par lequel les Pîrzâde s'occupent à chaque visite de leurs «invités » à qui ils servent d'intermédiaires, d' " avocats » auprès du saint ; et dont ils reçoivent des dons en retour. Ils organisent les concerts de qawwâlî : l'argent donné par les auditeurs à l'intention des musiciens passe d'abord par leur main et ils en contrôlent la répartition. Ils ont aussi le monopole des autres activités à incidence lucrative qui sont soit spirituelles comme la direction et l'initiation des disciples des fidèles et le traitement des malades à l'aide d'exorcismes et d'amulettes, soit purement commerciales comme la vente des fleurs et de livres et gravures. Ils possèdent donc un double contrôle spirituel et matériel sur le dargâh. Ils se conduisent en maîtres à la fois par rapport à leurs propres femmes reléguées hors de l'espace public, dont nous parlerons plus loin, et par rapport aux groupes socialement infériorisés qui assurent un service visible dans le dargâh, comme nous allons le voir tout de suite. Ces derniers peuvent être répartis en deux classes distinctes : les gens de service et les mendiants.

Parmi les gens de service, ceux qui ont sans doute le plus bas statut, mais qui sont les plus importants, car indispensables à

26. Marc Gaborieau, Ni Brahmanes ni ancêtres. Colporteurs musulmans du Népal, Nanterre, Société d'ethnologie, 1993, p. 374-380. 
l'activité mystique, sont les musiciens ou qawwâl. En principe illettrés, ils sont les dépositaires et les exécutants d'un vaste répertoire transmis oralement ${ }^{27}$. Il ne s'ensuit pas, pour autant, qu'ils soient haut placés : dans la civilisation indienne comme dans le monde islamique, les musiciens professionnels, même s'ils remplissent une fonction religieuse, sont infériorisés et rangés parmi les plus basses castes intouchables. Dans les sanctuaires soufis, il n'en va guère mieux : visitant un jour Nizamuddin avec un universitaire musulman ethnologue célèbre, je lui demandai quel rang il attribuait aux qawwâl ; il les rangea sans hésiter parmi les Bhangî, balayeurs, c'est-à-dire la plus basse des castes intouchables. Même s'il nous manque une enquête ethnologique détaillée sur la place exacte de ces musiciens dans la hiérarchie, il est sûr qu'il s'agit d'une très basse caste. Ils ont leur maison dans le dargâh même, à la périphérie des habitations des Pîrzâde à qui ils sont liés héréditairement.

Probablement de statut légèrement supérieur sont les porteurs d'eau, qui ont le surnom de " célestes », bahishtî, dans cette contrée subtropicale. Même s'ils ont perdu beaucoup de leur utilité avec l'installation de l'eau courante, ils sont présents avec leurs outres en peau de chèvre lors des fêtes. On signale aussi d'autres petits métiers à l'usage des pèlerins, comme les «éventeurs » qui les rafraîchissent avec de grands éventails de toile emmanchés sur une longue perche. La cuisine charitable, le langar, emploie aussi de nombreux serviteurs.

Les mendiants peuvent se répartir en deux catégories. D'abord ceux qui ont une identité religieuse : les Fakirs (faqîr) qui sont en quelque sorte des soufis «de basse caste », nettement subordonnés aux Pîrzâde et qui mendient à l'entrée du dargâh en échange ou non de quelques bénédictions. Chaque région du sous-continent indien a ses propres confréries de Fakirs infériorisés : ceux qui hantent Nizamuddin, notamment au moment des fêtes, n'ont pas été clairement identifiés. Tous ces Fakirs sont traditionnellement classés

27. Le répertoire du principal chanteur de Nizammudin vient pour la première fois d'être fixé par écrit: Mi'râj Ahmad Qawwâl, Surûd-i ruhânî. Qawwâlî-ke rang, edited with an introduction by Claire Devos, DelhiNizamuddin, Ghulam Hasnain, 1998. 
comme « hétérodoxe », be-shar', c'est-à-dire n'observant pas la Loi divine. Comme je l'ai amplement démontré ailleurs ${ }^{28}$, cette expression spécifiquement indienne équivaut à l'arabe malâmatî, désignant celui qui recherche le blâme en s'écartant de la Loi ; elle est appliquée à ces soufis de seconde zone parce qu'on leur impute des manquements (vrais ou supposés) aux injonctions de l'islam. En fait cette condamnation morale masque le vrai fondement de la distinction, qui est hiérarchique : tous ces soufis de seconde zone, réduits à mendier et à effectuer des exploits fakiriques, sont dans les sanctuaires en position de subordination par rapport aux grandes confréries respectables. Il n'est pas exagéré de les appeler des soufis de basse caste.

D'autres mendiants, qui hantent le dargâh notamment au moment de la distribution des repas du langar et des fêtes n'ont pas d'identité religieuse. Mais ils ont une fonction religieuse certaine : en acceptant les aumônes, ils permettent aux pèlerins de gagner des mérites. Ils sont d'origine diverse géographiquement et socialement : veuves, orphelins, ratés, infirmes, malades, désaxés ou carrément fous, ils constituent la cour des miracles qui donne à Nizamuddin cet aspect médiéval qui en fait le charme pour les touristes s'ils n'ont pas peur d'affronter la promiscuité, voire la saleté... sans compter la boue en temps de pluie.

On a donc une gradation de statuts nettement distincts. Il n'y a pas d'études permettant d'établir avec précision le statut respectif de ces différents groupes; mais par extrapolation à partir de ce que nous savons sur la société musulmane de l'Inde du Nord, on peut inférer que l'ordre hiérarchique décroissant doit être quelque chose comme : Pîrzâde, gens de services respectables (porteurs d'eau, éventeurs...), Fakirs, Qawwâls ; la population flottante des mendiants autres que les Fakirs, dont les origines sont mal connues, n'a pas de statut défini. L'adjectif « féodal » n'est pas assez fort pour caractériser cet ordre. Il s'agit d'un échantillon représentatif de la société

28. Marc Gaborieau, «Le concept de malâmat en Inde : hétéropraxie et hiérarchie », dans Nathalie Clayer, Alexandre Popovic \& Thierry Zarcone, Melamis-Bayramis. Études sur trois mouvements mystiques musulmans, Istanbul, Éditions Isis, 1998, p. 37-49. 
musulmane de l'Inde qui, comme j'en ai fait la démonstration ailleurs ${ }^{29}$, est une société de castes.

\section{« Grenouilles au fond d'un puits »: subordination des femmes}

Une autre forme de subordination, interne cette fois à chaque groupe de statut, est celle des femmes aux hommes. Elle est particulièrement marquée chez les Pîrzâde. Sa manifestation la plus remarquable est la stricte ségrégation de leurs femmes, qui n'entrent jamais dans l'espace public du sanctuaire : elles ne viennent même pas prier sur la tombe. Si elles doivent exceptionnellement sortir de leur maison, elles seront entièrement recouvertes de la burqa $a^{`}$ que les événements d'Afghanistan ont rendue célèbre. On a la chance d'avoir une remarquable monographie ethnologique sur elles, faite par une femme qui seule pouvait entrer dans ce monde totalement fermé à l'observateur masculin ${ }^{30}$. Elles sont subordonnées aux hommes dans la sphère domestique d'abord, devant cuisiner pour la famille, procréer et élever les enfants, qui perpétueront le lignage si ce sont des garçons, et serviront de monnaie d'échange pour les alliances, si ce sont des filles. Ces femmes ont aussi un rôle certain, mais de second plan, dans l'économie du dargâh. Sur le plan rituel d'abord : elles confectionnent des objets pour les rites, notamment les draps brodés qui recouvrent la tombe et des pâtisseries à bénir et distribuer comme tabarruk lors des fêtes. Sur le plan social ensuite : elles doivent organiser l'hospitalité et la nourriture pour les « invités » les plus généreux de leurs maris, qui seront logés dans leur maison pour encourager un flot continu de donations. Leur rôle est donc indispensable à la bonne marche du sanctuaire.

Les musiciens, par un processus tout à fait classique d'imitation de leurs maîtres Pîrzâde, voilent aussi leurs femmes.

\section{Hiérarchie et contestation : l'art de faire des scènes}

Cette hiérarchie des castes et des genres produit-elle un monde harmonieux où l'autorité est suffisamment assurée pour éviter tout

29. Marc Gaborieau, Ni brahmanes ni ancêtres..., op. cit., chap. IX.

30. Patricia Jeffery, Frogs in a Well..., op. cit. 
conflit ? Certaines études, même récentes, le donneraient à penser ${ }^{31}$. Il est pourtant bien connu que les conflits sont de règle dans les sanctuaires soufis pour des enjeux de prestige et de finances : les minutes des procès intentés en vue de s'assurer le leadership constituent d'importantes archives pour l'histoire de ces institutions ${ }^{32}$.

À quel niveau se nouent ces conflits? Dans la société indienne telle qu'elle était au moment où ont été faites les principales études il y a quelque vingt-cinq ans - c'est-à-dire avant la montée des revendications féministes et le passage au politique des basses castes - ni les gens de service, ni les femmes n'avaient les moyens de se révolter; encore moins les Fakirs et les mendiants. Les conflits se jouaient au plus haut niveau, là où les enjeux de prestige et de finance sont les plus élevés, c'est-à-dire chez les Pîrzâde qui se partagent les profits du dargâh. Le système prétendument juste qui veut un partage égalitaire des ressources entre les lignages a des effets pervers, car aucun ne supporte d'être placé au même niveau que les autres et recherche la distinction. Le seul moyen pacifique d'instaurer une telle distinction est de s'arranger pour avoir une clientèle de disciples et d' «invités » plus importante que les concurrents. Quand cela ne suffit pas, la solution pour réaffirmer les préséances est généralement recherchée dans les esclandres publics devant la foule des pèlerins au moment des fêtes : c'est l'art de « faire des scènes »-finement analysé par Patricia Jeffery - qui vise à changer la distribution des atouts entre les clans en compétition. Jeu théâtral mais un peu vain, car de toute façon les Pirzâde sont trop nombreux pour des revenus trop maigres.

\section{CONClusion}

Au terme de cette analyse, le sanctuaire de Nizamuddin, devenu centre de pèlerinage parce qu'il est marqué par la baraka d'un saint, apparaît comme le lieu où s'opère une double médiation dans

31. Soren Lassen, "A meeting Place of Traditions...", op. cit.

32. Voir par exemple Richard M. Eaton, "Court of Man, Court of God. Local Perceptions of the Shrine of Bâbâ Farîd...", op. cit. 
la marche du pèlerin vers Dieu. Le saint lui-même, de sa tombe, est le premier médiateur : il est l'intercesseur qui obtient pour les fidèles les faveurs sollicitées dans les rites ; sa spiritualité domine l'initiation mystique et la musique extatique. Mais entre le pèlerin et le saint, une nouvelle médiation intervient : elle est assurée éminemment par les desservants du temple, les Pîrzâde, descendants et substituts du saint, qui supervisent à leur profit l'exécution des rites, l'initiation mystique et les concerts spirituels; les gens de service comme les musiciens, et les mendiants comme les fakirs ne sont que les auxiliaires de cette seconde médiation.

Dans quelle mesure Nizâmuddin est-il représentatif des sanctuaires soufis de l'Inde ? En lisant les monographies qui se sont succédé sur la question, on retrouve partout les mêmes éléments essentiels qui contribuent à créer une microsociété hiérarchisée qui sert et exploite à la fois les pèlerins. Seuls deux seuls traits différencient nettement Nizamuddin des autres : le partage démocratique des tâches et des revenus entre les familles des quatre clans héritiers du saint ; et l'absence de dotation en terres assurant un revenu régulier. Ailleurs, dans les dargâh largement pourvus de terres, la structure du pouvoir est généralement monarchique : à chaque génération un seul des descendants du saint, est désigné comme son héritier et comme maître absolu du sanctuaire et de ses revenus : c'est le sajjâdâ-nashîn, " celui qui est assis sur le tapis de prière (du saint fondateur) ». Les conflits de pouvoir portent alors sur la désignation $\mathrm{du}$ « monarque » qui siégera sur ce trône, les prétendants malheureux n'hésitant pas à recourir aux tribunaux. Deux cas exemplaires ont été récemment étudiés. Le premier est celui du dargâh de Bâbâ Farîd (1175-1265), le troisième grand Chishti, à Pak-Pattan, aujourd'hui au Pakistan ${ }^{33}$. Les desservants, eux aussi descendants du saint, sont de riches propriétaires fonciers qui comptent parmi les grands notables de la région; ils ont même pu, durant les troubles du XVIII ${ }^{\mathrm{e}}$ siècle, s'ériger en principauté autonome. L'autre exemple est indien : c'est celui du dargâh de Salon dans le district de Rai

33. Richard M. Eaton, "Court of Man, Court of God. Local Perceptions of the Shrine of Bâbâ Farîd...", op. cit. ; Richard M. Eaton, "The Political and Religious Authority of the Shrine of Bâbâ Farîd", op. cit. 
Bareilly près de Luchnow ${ }^{34}$. Relevant aussi de la Chishtiyya, il fut fondé au début XVII par Shâh Pîr Muhammad Salonî (1586-1787), disciple de Shâh 'Abd al-Karîm Manikpûrî (m. 1647/8). Il s'agissait, comme dans le cas de Pak-Pattan, d'un sanctuaire doté de grandes propriétés foncières (44 villages !) depuis l'empereur Aurangzeb dans la seconde moitié du XVII ${ }^{\mathrm{e}}$ siècle. Le sajjâda-nashîn vivait sur le pied d'un grand propriétaire, voire d'un Nawwâb; ses dévots, hindous aussi bien que musulmans, étaient aussi souvent ses métayers. Les fêtes étaient des plus grandioses (avec une assemblée distincte pour les femmes, avec des musiciens femmes) et une grande prédilection pour les rites coutumiers souvent d'origine hindoue. Le sajjâda-nishîn y tenait une espèce de cour où il instruisait toutes les requêtes possibles. Il avait le train de vie d'un grand propriétaire foncier dont il avait tous les attributs : costume, chasse, danseuses pour les cérémonies du cycle de vie... D'une façon générale, les maîtres des grands sanctuaires soufis appartiennent à l'aristocratie foncière: il est symptomatique qu'au Pakistan le terme murîd signifie à la fois disciple et métayer. Il arrive cependant que le pouvoir de ces chefs de dargâh soit encadré par des administrateurs, mutawallî, nommé par le pouvoir politique, comme ce fut le cas dès l'époque moghole dans le plus vieux sanctuaire Chishtî de l'Inde, celui d'Ajmer ${ }^{35}$. Pauvres ou richement dotés, en plus des fonctions religieuses que nous avons longuement analysées, ces sanctuaires remplissent un double rôle culturel. Ils servent d'un côté à préserver et à répandre la culture indo-persane, et plus récemment ourdoue, qui est celle de l'islam indien. Nizâmuddin est exemplaire sur ce point : rappelons que c'est Nizâmu'd-Dîn et Amîr Khusrau, enterrés là, qui ont fixé les plus hauts standards de l'hagiographie et de la poésie indo-persane qui ont essaimé dès la seconde moitié du XIV sur l'ensemble du sous-continent comme l'a souligné une étude récente ${ }^{36}$. Le plus grand poète de l'Inde

34. Claudia Liebeskind, Piety on its knees..., op. cit., p. 125-176.

35. P. M. Currie, The Shrine and Cult of Mu'in al-Dîn Chishtî..., op. cit., p. $155,164-173$.

36. Simon Digby, "Before Timur came: Provincialization of the Delhi Sultanate through the Fourteenth Century", Journal of the Social and Economic History of the Orient, vol. 47, n 3, 2004, p. 298-356. 
moderne, Mîrzâ Ghalîb (1796-1869), réputé pour ses compositions en persan et en ourdou, y est aussi enterré : dans la Ghalib Academy qui jouxte sa tombe se tiennent chaque année des joutes poétiques. Principal foyer de peuplement musulman à Delhi après les migrations massives vers le Pakistan qui ont suivi la partition de l'Inde, Nizamuddin est une sorte de conservatoire de la culture musulmane de l'Inde.

Les sanctuaires contribuent aussi à l'acculturation de l'islam. Les pèlerins hindous, $\mathrm{y}$ compris des femmes, $\mathrm{y}$ viennent nombreux. Les soufis ont eu surtout à cœur de reprendre de nombreux éléments de la culture indienne, acclimatant ainsi le soufisme à son nouvel habitat. Nous en avons noté de nombreux exemples : la circumambulation de la tombe se fait dans le sens des aiguilles d'une montre comme dans les temples hindous, et non dans le sens contraire comme dans le tawâf arabe autour de la Ka`ba et des tombes de saints ; la fête hindoue de printemps, basant, est célébrée à une date fixée selon le calendrier hindou ; la hiérarchie des groupes de statut dans le dargâh doit beaucoup au système des castes.

Le rôle politique de Nizamuddin ne s'est pas démenti, même depuis l'indépendance et la partition du sous-continent : à l'instar des sultans et des empereurs d'antan, les politiciens du Pakistan et du Bangladesh viennent chercher une légitimation dans les grands sanctuaires de la Chishtiyya, notamment à Nizamuddin qui est un must. En Inde, les politiciens hindous ne sont pas de reste: Indira Gandhi allait ostensiblement à Ajmer ; paradoxalement l'exPremier Ministre de l'Extrême Droite hindoue, qui faisait de son hostilité aux musulmans son fonds de commerce, s'y était aussi rendu.

Enfin le processus de mondialisation n'a pas épargné Nizamuddin. Entre les deux guerres, un musicien soufi qui y est enterré, Inâyat Khân (m. 1927), a popularisé la Chishtiyya en Europe et aux ÉtatsUnis $^{37}$; des Occidentaux se pressent toutes les semaines pour les

37. Carl W. Ernst \& Bruce B. Lawrence, Sufi Martyrs of Love..., op. cit., p. $140-143$. 
concerts de Qawwâlî exécutés sur sa tombe. Le centre du Tablîgh ${ }^{38}$, mouvement prosélyte transnational fondé précisément à Nizamuddin en 1927, et sa principale librairie s'y trouvent aussi : de jeunes musulmans militants du monde entier viennent aussi s'y ressourcer. Bien que ce mouvement dérivé de l'école de Deoband ait aujourd'hui pris ses distances avec la spiritualité soufie et le culte des saints, il n'a pas rompu le cordon ombilical qui le rattache, par son fondateur qui y est enterré, au sanctuaire soufi le plus vivant de l'Inde.

5 rue Neuve Saint Germain

92100 Boulogne-Billancourt (France)

mgb@ehess.fr

38. Marc Gaborieau, «Renouveau de l'islam ou stratégie politique occulte ? La Tablîghî Jamâ'at dans le sous-continent indien et dans le monde », dans Catherine Cémentin-Ojha (éd.), Renouveaux religieux en Asie, Paris, École Française d'Extrême Orient, p. 211-229; Marc Gaborieau, Article "Tablîghî Djamâ'at", Encyclopédie de l'islam, 2e éd., vol. X, 1998, p. 39-40 ; Muhammad Khalid Masud (éd.), Travellers in Faith. Studies of the Tablîghî Jamâ 'at as a Transnational Islamic Faith Movement for Faith Renewal, Leyde, Brill, 2000. 


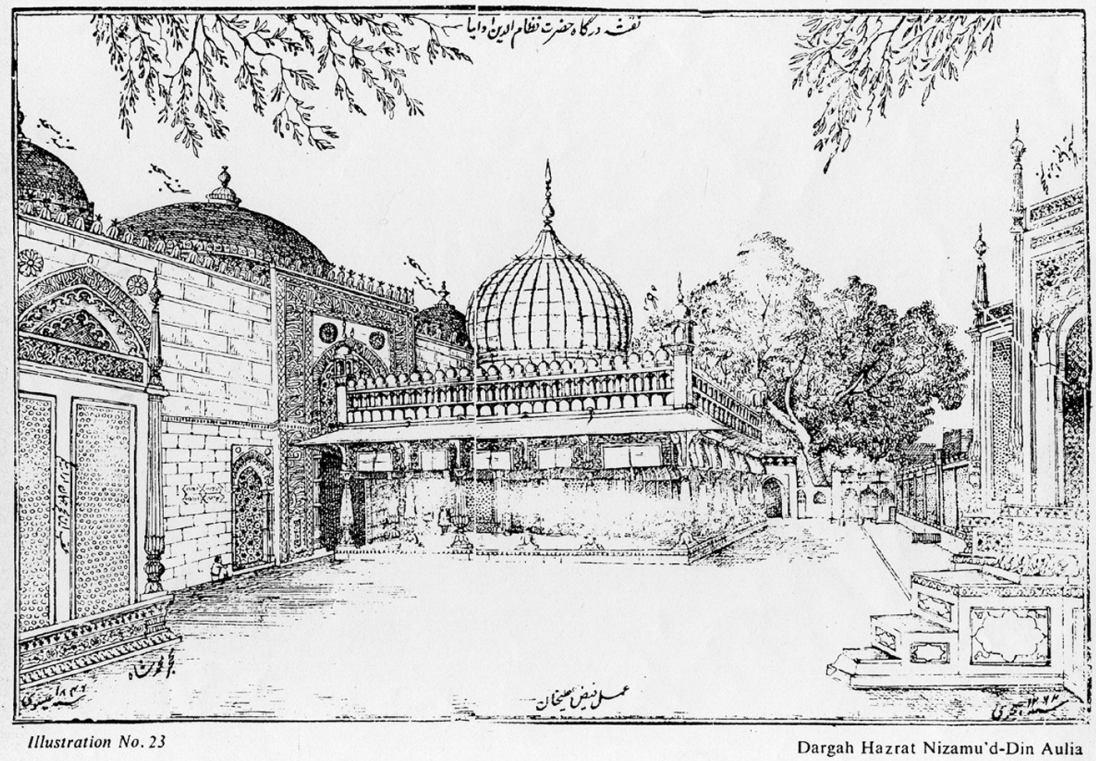

Gravure sur bois représentant le dargâh de Nizâmu'd-Dîn parue à Delhi en 1846 dans la description des monuments de Delhi publiée en ourdou par Sayyid Ahmad Khân (1817-1898) sous le titre Athâru's-Sanâdid. La gravure est datée 1846, signée Faiz 'Alî Khân. Des inscriptions en ourdou sur la gravure identifient les monuments représentés : au centre, le mausolée (dargâh); à gauche, la mosquée avec ses trois dômes; au premier plan à gauche, l'enclos contenant les tombes de la princesse moghole Jahân Ârâ (1614-1681) et de l'empereur moghol Muhammad Shâh (1719-1748); au premier plan à droite, la tombe marquée comme celle de Mirzâ Jahângîr - pour Atgah Khân (mort en 1662) ou son fils Mirzâ 'Azîz Koka (c. 15421624), tout deux enterrés à l'est du mausolée. 


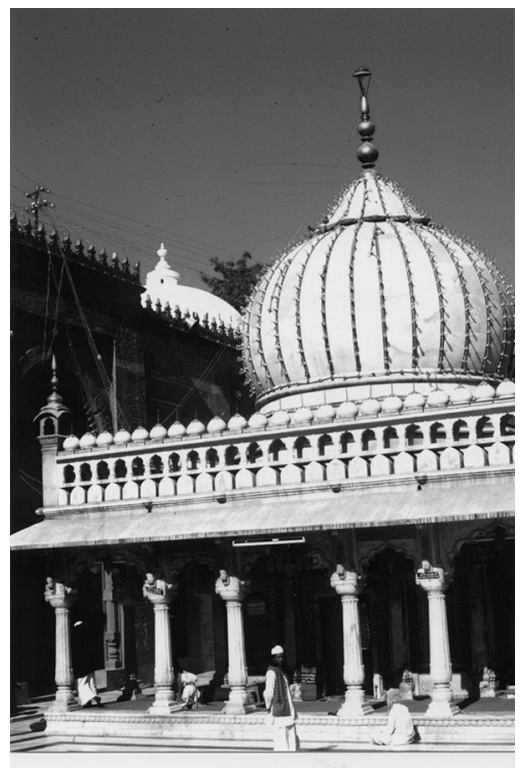

Le mausolée de Nizâmu'd-Dîn (en décembre 1975), façade sud, avec un desservant debout devant la porte d'entrée, attendant les pèlerins, et des fidèles sous la véranda; sur la gauche, la mosquée du XIV e siècle en grès, avec l'un de ses dômes peint en blanc. (Cliché Marc Gaborieau)

Sur l'espace dégagé devant le mausolée de Nizâmu'd-Dîn (en décembre 1975), côté sud, deux chanteurs (qawwâl) s'accompagnant d'un harmonium (au fond) et d'un tambour (au premier plan). Au fond, l'arche méridionale de la mosquée. À gauche, l'enclos en marbre de la tombe de Jahân Ârâ. (Cliché Marc Gaborieau)

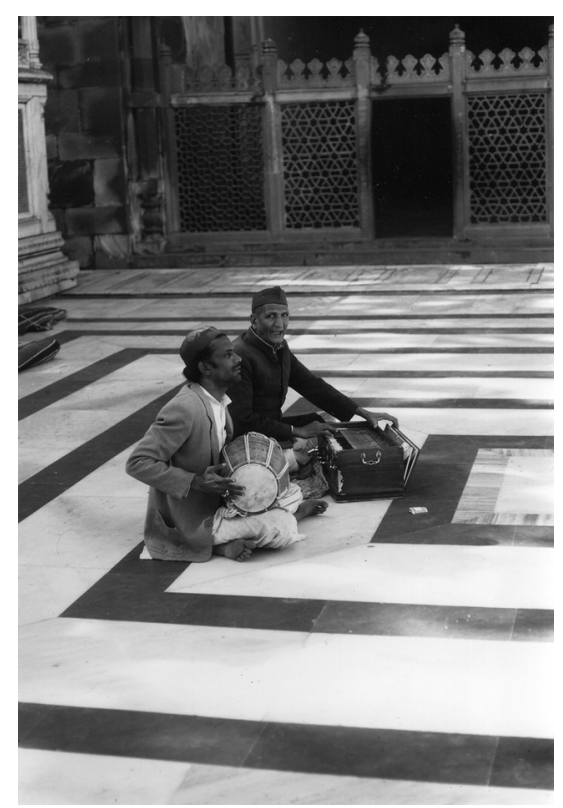


